BULLETIN Bulletin hispanique

HISPANIQUE Université Michel de Montaigne Bordeaux

112-1| 2010

Actes du Colloque « langue, littérature, littéralité »

\title{
De la littéralité aux fragments
}

\section{Milagros Ezquerro}

\section{(2) OpenEdition}

Journals

Édition électronique

URL : http://journals.openedition.org/bulletinhispanique/1189

DOI : 10.4000/bulletinhispanique.1189

ISSN : 1775-3821

\section{Éditeur}

Presses universitaires de Bordeaux

\section{Édition imprimée}

Date de publication : 1 juin 2010

Pagination : 433-439

ISBN : 978-2-86781-692-5

ISSN : 0007-4640

Référence électronique

Milagros Ezquerro, « De la littéralité aux fragments », Bulletin hispanique [En ligne], 112-1 | 2010, mis en ligne le 01 juin 2013, consulté le 04 mai 2019. URL : http://journals.openedition.org/ bulletinhispanique/1189; DOI : 10.4000/bulletinhispanique.1189 


\title{
De la littéralité aux fragments
}

\author{
Milagros EzQuerro \\ Université de Paris - Sorbonne Paris IV
}

Après une analyse des notions les plus importantes de l'article de Nadine Ly, La littéralité, on les confronte à la théorie du texte proposée par Milagros Ezquerro dans Fragments sur le texte, en soulignant les convergences et les divergences principales des deux approches de l'analyse des textes, élaborées à la même époque.

Después de un análisis de las nociones más importantes del artículo de Nadine Ly, La littéralité, se cotejan con la teoría propuesta por Milagros Ezquerro en Fragments sur le texte, subrayándose las convergencias y las divergencias principales de los dos acercamientos al análisis de los textos, elaborados en la misma época.

This essay will begin by an analysis of the most important notions in Mrs Ly's article on "literality", followed by a comparison with the theory of text put forward by M. Ezquerro in 'Fragments sur le texte'. It ultimately will underline the main points of convergence or divergence between the two approaches of textual analysis that were elaborated at the same period.

Mots-clés : Analyse textuelle - Littéralité - Théorie du texte.

C EST EN 1989 - vingt ans déjà, mais "Que veinte años no es nada " affirme le tango - que le Groupe Interdisciplinaire d'Analyse Littérale (GRIAL, composante de l'URA 1047 du CNRS) publie le premier volume d'études collectives sur la Littéralité. Ce volume était le fruit du

$B H i$, Tome 112, no 1 - juin 2010 - p. 433 à 439 
travail d'un groupe de réflexion qui s'était constitué quelques années plus tôt autour de l'analyse textuelle littérale, et qui rassemblait un certain nombre de personnes présentes à ce Colloque Hommage à Nadine Ly. Littéralité 1 offre une série d'analyses sur la double pièce attribuée à Calderón, $L a$ hija del aire, de Pierre Heugas, Frédéric Bravo, Marie Aranda, Nadine Ly et Béatrice Chenot, auxquelles s'ajoutent une étude de l'ouverture de Si te dicen que caí de Juan Marsé par Geneviève Champeau et, en annexe, une réflexion de Nadine Ly sur le concept de Littéralité, qui constitue une sorte de Manifeste du groupe. De ce texte, qui avait été écrit pour le IVe Colloque de Psychosystématique du langage, tenu à Cerisy-La Salle en juin 1986, je citerai un extrait particulièrement significatif :

La littéralité du texte est donc le lieu où la matérialité et la théorie du langage, où la matérialité et la théorie de l'écriture s'érigent en système signifiant. Car c'est bien de système qu'il s'agit : aussi court ou aussi long qu'on voudra l'envisager (du vers ou de la phrase à la strophe et au chapitre, ou au recueil de poèmes et au roman, ou encore à l'œuvre complète), chaque texte forme un système clos, intangible, dont la matérialité non dégradable (je ne parle pas ici de transmission orale), s'offre au regard et à l'observation, identique et toujours une - les variantes elles-mêmes, invariables dans leur fluctuation figée, fixée, constituant un élément de cette identité ${ }^{1}$.

De cet énoncé programmatique, je retiendrai quelques notions essentielles :

- Le texte, quelle que soit son extension, est un système signifiant,

- Chaque texte forme un système clos, intangible,

- La matérialité du texte s'offre à l'observation identique et toujours une.

Il convient aussi de souligner que le GRIAL, dès ses premières réunions, a travaillésimultanémenten pratiquantl'analysedestextesàdesfinspédagogiques, et en théorisant cette pratique dans le contexte d'effervescence sémiologique, propre aux années soixante-dix et quatre-vingts. La même démarche était suivie, dès 1975, par le SEL (Séminaire d'Études Littéraires) de Toulouse, dont les premiers colloques ${ }^{2}$, très artisanaux à une époque où les équipes de

1. Nadine Ly, "La Littéralité », in Littéralité 1, Université de Bordeaux III - CNRS, 1989, p. 194.

2. 1977 : "Sujet et sujet parlant dans le texte", 1978 : «L'idéologique dans le texte ", 
recherche universitaires n'avaient pas d'existence légale, avaient réuni la fine fleur du nouvel hispanisme. Ces démarches créatrices étaient dans l'air du temps, d'un temps "post soixante-huitard ", aujourd'hui décrié par d'aucuns, où l'obsession n'était pas d'évaluer, mais d'inventer. Nous avions en outre - on ne saurait trop le rappeler - une figure tutélaire, un exemple et un maître en la personne de Maurice Molho, dont la parole et l'écriture sont, pour beaucoup d'entre nous, à la source de notre pratique enseignante et de nos écritures, pourtant si diverses.

La pratique de l'analyse textuelle que les membres du GRIAL, autour de Nadine Ly, baptisèrent du beau nom de Littéralité, était fondée sur une attention extrême portée à la matérialité du texte dans tous ses aspects : phonique, lexicale, rythmique, syntaxique, rhétorique. Il n'est que de lire les articles réunis dans ce volume pour apprécier la productivité de cette approche du texte. S'agissant de la double pièce dramatique de Calderón, on peut voir que la Littéralité n'affecte pas seulement le langage poétique et les métaphores, qui sont un terrain de choix pour mettre en valeur l'extraordinaire prégnance du signifiant, mais aussi la structure dramatique, la construction et le fonctionnement des personnages, et, en dernière instance, l'idéologie du drame caldéronien. En effet, il ne faut pas s'y tromper, construire une pratique de l'analyse textuelle est loin d'être un acte anodin, un acte pédagogique faible, c'est au contraire poser une vision du texte qui s'oppose à d'autres pratiques, à d'autres visions, c'est un acte idéologique fort parce qu'il s'appuie sur une théorie du langage qui ne saurait être neutre. C'est ainsi que, au moment de définir les fondements de la Littéralité, Nadine Ly est obligée de marquer la différence entre cette approche du texte et d'autres, couramment pratiquées à cette époque :

C'est pourquoi je m'inscris en faux contre toute analyse du texte conçu comme chaîne de substitutions entre un hypothétique et fluctuant (y) qu'on attendait de préférence à un $(\mathrm{x})$ déroutant. La lettre du texte c'est bien (x) et non (y), quelles que soient par ailleurs les ressemblances phoniques ou sémantiques, les ricochets associatifs, qui rapprochent du trop présent (x) le si confortable (y) que pourtant la littéralité refuse et, le refusant, annule et interdit.

1979 : «L'œuvre de Augusto Roa Bastos et la réalité paraguayenne : problèmes méthodologiques ",

1980 : " Organizaciones textuales ",

1982 : "Los escritores hispanoamericanos frente a sus críticos",

1983 : "Le personnage en question",

1985 : "Teoría del discurso poético ». 
Hors du texte, point de salut : telle est la loi de la littéralité, celle à laquelle j'ai décidé de soumettre mes analyses textuelles, quoi qu'il puisse en coûter d'interprétations et de rêveries sacrifiées ${ }^{3}$.

Il y a quelque chose d'ascétique dans cette posture, un respect de la lettre, que l'on pourrait qualifier de religieux, ou alors de fétichiste, et, par ailleurs, un renoncement aux facilités de l'interprétation molle, aux délices des rêveries hédonistes sur un texte fluctuant au gré des humeurs du lecteur. Pourtant, la créativité de cette approche des textes est très évidente à la simple lecture de ses applications. Il ne s'agit pas, loin de là, d'une simple description de la matérialité du texte, puisque la phase descriptive est aussitôt complétée par l'interprétation des caractéristiques relevées. Le but est bien de dégager du sens, tout le sens qu'on peut tirer de la description des divers signifiants qui conforment le texte. En cela, la Littéralité se démarque aussi d'une démarche purement descriptive à laquelle pourrait mener un structuralisme mal compris, et qui est la grande tentation de nos étudiants, timides devant l'engagement personnel que suppose toute interprétation, pour aussi fondée qu'elle soit sur une minutieuse description. Ici se pose, bien évidemment, la question de la réception et du sujet récepteur, sur lesquels il nous faudra revenir.

À la même époque où Nadine Ly et le GRIAL travaillaient sur le concept de Littéralité, je réfléchissais, avec le SEL, sur une théorie du texte, qui s'est élaborée lentement jusqu'à prendre forme dans un petit ouvrage que j'ai finalement intitulé, en hommage à Roland Barthes et à ses Fragments d'un discours amoureux, Fragments sur le texte ${ }^{4}$. C'est en 1984, lors d'un Symposium de Théorie Sémiotique organisé à Madrid par le Consejo Superior de Investigaciones Científicas, que j'ai présenté le premier exemple d'application de cette théorie en formation à une notion qui nous intéressait beaucoup, celle du narrateur ${ }^{5}$ ou, plus exactement, de la fonction narratrice. Je présentais alors la notion de "système complexe ouvert ", empruntée aux sciences de la vie, notion très féconde si on l'applique au texte, et que je voudrais confronter avec celle de "système signifiant clos et intangible " proposée par la Littéralité. Bien évidemment, la notion de "système signifiant complexe " est commune aux deux théories : en effet, non seulement le texte est composé d'éléments multiples imbriqués à plusieurs

3. N. Ly, «La Littéralité », in Littéralité 1, op. cit., p. 195.

4. Milagros Ezquerro, Fragments sur le texte, Paris, L'Harmattan, coll. «Langue \& Parole ", 2002.

5. M. Ezquerro, "La función narradora como sistema complejo abierto", in Teoría semiótica. Lenguajes y textos hispánicos, Volumen I, Madrid, Consejo Superior de Investigaciones Científicas, 1985, p. 517-522. 


\section{DE LA LITTÉRALITÉ AUX FRAGMENTS}

niveaux, mais encore, en tant que totalité, il est plus que la somme des éléments qui le composent : "La Littéralité dit un sens qui n'est pas la somme des sens partiels construits par les éléments du texte mais plutôt leur sommation. " Là où les deux théories divergent, c'est au niveau de la clôture du texte, revendiquée par la Littéralité. Portés par la pensée biologiste, les Fragments théorisent un texte conçu sur le modèle de l'organisme vivant, qui se constitue comme un tout, comme un système apparemment clos sur lui-même, mais en réalité toujours en interaction avec son biotope, avec le milieu ambiant dans lequel il vit.

Le texte est aussi un système ouvert puisqu'il est inévitablement inscrit dans un contexte, et même dans des contextes différents selon les diverses étapes du processus qui le constitue. Dans la phase de production, le texte est relié au contexte du producteur (idiotope A) qui est lui-même un système complexe ouvert puisqu'il est composé de multiples éléments (données biographiques, socio-historiques, cognitives et psychologiques du producteur, circonstances intérieures et extérieures de la production) qui, à leur tour, sont connectés à d'autres systèmes complexes ouverts. Dans la phase d'observation, le texte est relié, en plus, au contexte de l'observateur (idiotope $\Omega$ ) qui présente des caractéristiques de même nature. Ces trois systèmes complexes ouverts fonctionnent en réseau et échangent continuellement entre eux de grandes quantités d'informations : texte et contextes sont donc en interaction permanente, ils se modifient mutuellement dans des proportions très variables ${ }^{6}$.

En réalité, je sais pertinemment que le terme " clos » appliqué au texte ne signifie nullement - et les analyses présentées le montrent parfaitement que la Littéralité ne suppose pas une circulation d'informations entre les trois systèmes (celui de l'auteur, celui du lecteur et celui du texte), simplement elle la conçoit autrement :

La Littéralité c'est le même du texte, c'est le texte propre, constamment offert à l'autre du regard porté sur lui, à l'innombrable et variable lecteur ; un même qui s'est par ailleurs définitivement détaché et libéré de l'Autre, de l'Auteur qui par son nom en assume la responsabilité, et de cet autre plus diffus qui l'a permis et produit : époque et lieu, conditions politiques, socio-économiques, conditions mentales, biographiques, conscientes et inconscientes, linguistiques, rhétoriques, « littéraires » etc... ${ }^{7}$

6. M. Ezquerro, Fragments, op. cit., p. 95.

7. N. Ly, «La Littéralité », in Littéralité 1, op. cit., p. 194. 
C'est une conception plus poétique, celle d'un objet perçu dans son ipséité, libéré des contingences de sa production, et offert au regard de l'observateur sans que celui-ci puisse en rien l'entamer ou l'offusquer. La conception du texte que proposent les Fragments est celle d'un objet fortement sollicité par ses contextes, en perpétuelle mutation tout au long de son histoire, livré aux exactions de chaque lecteur, sans que pour autant son corps propre en soit réellement affecté. Car la propriété la plus singulière du texte est sa créativité, due à ce qu'Henri Atlan appelle un "phénomène d'auto-organisation " : en sciences de l'information, on qualifie ainsi des systèmes capables de créer des significations nouvelles :

Il s'agit de concevoir des modèles d'organisation capables de se modifier eux-mêmes et de créer des significations imprévues et surprenantes même pour le concepteur. La solution de ces paradoxes se trouve dans l'utilisation simultanée de deux ingrédients qui sont habituellement négligés dans la fabrication des modèles informatiques classiques : d'une part, une certaine quantité d'indétermination, de hasard dans l'évolution du modèle qui permet à du nouveau, non déterminé par le programme, de se produire ; d'autre part la prise en compte du rôle de l'observateur et du contexte dans la définition de la signification de l'information, grâce à quoi le nouveau, l'inattendu peuvent acquérir une signification et ne sont pas que du chaos et des perturbations aléatoires ${ }^{8}$.

On voit parfaitement comment un texte, conçu comme système complexe ouvert et auto-orgnisateur, peut créer, pour l'observateur, des significations nouvelles, imprévues et imprévisibles pour le producteur lui-même. L'indétermination, dans le domaine textuel, est multiple : c'est d'abord celle de la langue, dont le texte hérite et qu'il multiplie. C'est ensuite l'interaction entre texte et contextes divers, qui augmente avec le temps et le nombre de lecteurs. Quant au rôle du lecteur, il est considérable, on le sait, même si, par révérence envers la sacralité du texte, on a tendance à le sous-estimer. Curieusement, il nous semble toujours que donner de l'importance à la fonction du lecteur revient à en retirer à la fonction de l'auteur, dont la figure - quoi qu'on ait pu dire de « la mort de l'auteur » - garde l'aura que lui avait conférée le Romantisme. Pourtant, dans les années soixante-dix, l'École de Genève avec Hans Robert Jauss, dont Pour une esthétique de la réception, publié en 1975 et traduit en français en 1978, et Wolfgang Iser,

8. Henri Atlan, À tort et à raison. Intercritique de la science et du mythe, Paris, Seuil, coll. "Science ouverte ", 1986, p. 70. 
dont L'acte de lecture. Théorie de l'effet esthétique, publié en 1976 et traduit vingt ans plus tard en français, avaient mis en avant le rôle créateur de la réception. Et encore avant, deux grands romans latino-américains avaient fictionnalisé la figure du lecteur comme co-créateur de l'œuvre littéraire, je veux parler de Rayuela et de Yo el Supremo, respectivement publiés en 1973 et 1974, dans la ville de Borges, bien entendu. Malgré tout, la critique universitaire en France était bien plus intéressée par l'auteur et par le texte, c'est-à-dire par le processus de production, que par ce qu'il advient dans le processus de réception. Il semble bien que la Littéralité, au moins à ses débuts, ait focalisé son attention sur le texte en tant que système signifiant, reléguant au second plan ses attaches avec les deux sujets qu'il implique, même si elle ne les ignore pas. Sans doute le fait-elle par méfiance envers les pratiques analytiques (très répandues à l'époque dans l'hispanisme) qui donnaient une prééminence, lourde de conséquences, à l'auteur en tant que personne, à sa biographie, à ses engagements, ainsi qu'aux circonstances de l'écriture de l'œuvre. D'ailleurs, le Séminaire d'Études Littéraires partageait les mêmes préoccupations, comme le montrent les titres de ses premiers colloques : "Sujet et sujet parlant dans le texte ", "L'idéologique dans le texte ", "Organizaciones textuales ". Comme nous l'observions plus haut, proposer une théorie du texte et une pratique de l'analyse textuelle était - et sans doute continue-t-il à être - un acte engagé, sur le plan pédagogique et scientifique, et donc idéologiquement.

Je crois qu'aujourd'hui, à l'heure où l'Université est en butte aux assauts d'un pouvoir qui prétend ignorer ce que sont les enjeux de l'élaboration, de la transmission et de la mise en pratique des savoirs, afin de les formater au service d'une conception mercantile de la société, il convient que les Universitaires prennent conscience de ce qu'ils ont conquis, et qu'ils ne veulent pas perdre pour le présent et l'avenir. Il me semble que ce que Maurice Molho nous a légué de plus précieux c'est la liberté de penser et de dire. Mais cette liberté est aussi une injonction à réfléchir par soi-même, à ne pas accepter les opinions et les solutions prêtes à l'emploi, à remettre en question, à penser outre. C'est exactement ce qu'a fait Nadine Ly dans cette entreprise à la fois individuelle et collective que représente la Littéralité. C'est une entreprise d'élaboration, de transmission et de mise en pratique de savoirs reçus, hérités, thésaurisés, certes, mais aussi sans cesse remis sur le métier, réexaminés, critiqués, reélaborés en vue d'une nouvelle transmission, dont l'effectivité est tangible dans la présence ici de nouvelles générations, qui rend visible une filiation qui n'est pas celle du sang, mais celle du sens. 